\title{
COMPORTAMIENTO AGRONÓMICO Y PRODUCTIVO DE Tithonia diversifolia EN TRÓPICO ALTO BAJO DOS ESQUEMAS DE FERTILIZACIÓN
}

\author{
Agronomic and productive behavior of tithonia diversifolia in the high \\ tropics under two fertilization schemes
}

\section{Artículo de Investigación}

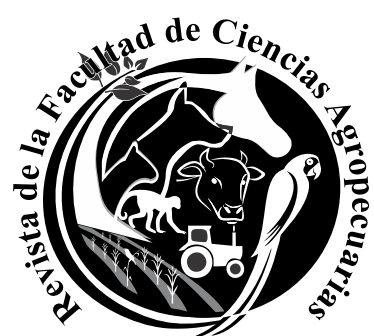

FAGROPEC

Recibido 21 de agosto de 2019. Aceptado 29 de noviembre de 2019.

${ }^{1}$ M.Sc. Zoot., Departamento de Zootecnia, Universidad de Pamplona, Colombia, Grupo de Investigación en Agricultura y Ganadería Sostenible GIAS

\section{https://orcid.org/0000-0002-3915-8396}

2.M.Sc. Zoot. Departamento de Zootecnia, Universidad de Pamplona, Colombia, Grupo de Investigación en Agricultura y Ganadería Sostenible GIAS.

(iD) https://orcid.org/0000-0002-0044-5566

Esp. Zoot. Departamento de Zootecnia, Universidad de Pamplona, Colombia, Grupo de Investigación en Agricultura y Ganadería Sostenible GIAS.

(iD https://orcid.org/0000-0003-0261-0542

4. Estudiante programa de Zootecnia, Universidad de Pamplona, Colombia, Grupo de Investigación en Agricultura y Ganadería Sostenible GIAS

*Autor para correspondencia: dixonfflorez@gmail.com
Dixon Fabián Flórez Delgado ${ }^{1^{*}}$, Alfonso Eugenio Capacho Mogollón ${ }^{2}$, Sandra Milena Quintero Muiño y Laura Patricia Ortiz Villamizar ${ }^{4}$

En Colombia, la Tithonia diversifolia, es uno de los recursos forrajeros más importantes en la alimentación animal, especialmente en lecherías especializadas y ganaderías doble propósito, gracias a su adaptación a variedad de climas, su productividad y valor nutricional. El objetivo de la presente investigación, fue evaluar el comportamiento agronómico y productivo de la Tithonia diversifolia bajo dos esquemas de fertilización. El estudio se llevó a cabo en el Centro de Investigación en Sanidad Vegetal y Bioinsumos CISVEB perteneciente de la Universidad de Pamplona. Para ello, se establecieron 50 plantas para cada tratamiento: fertilización química, fertilización orgánica y testigo bajo un diseño aleatorizado previo análisis de suelos y aplicación de correctivos. Para la evaluación de las variables agronómicas y productivas de altura de planta, número de tallos, número de hojas, peso de tallo, peso de hoja, relación hoja - tallo, área foliar y producción de materia seca, se aplicaron pruebas de estadística descriptiva, análisis de varianza y análisis de separación de medias mediante la prueba Tukey con una significancia del $5 \%$. Se presentó diferencia estadísticamente significativa $\mathrm{p}<0,05$ para las variables de altura, número de hojas y número de tallos, siendo el mejor tratamiento la fertilización química, mientras que área foliar fue superior en la fertilización orgánica. Para las demás variables se obtuvo comportamiento similar. Se concluye, que la fertilización química ejerce influencia sobre algunos parámetros agronómicos de la $T$. diversifolia.

Palabras claves:

Fertilización; Materia seca; Tithonia diversifolia; Variables agronómicas.

\begin{abstract}
In Colombia, Tithonia diversifolia is one of the most important forage resources in animal feed, especially in specialized dairies and dual-purpose livestock, thanks to its adaptation to a variety of climates, its productivity and nutritional value. The objective of the present investigation was to evaluate the agronomic and productive behavior of Tithonia diversifolia under two fertilization schemes. The study was carried out at the CISVEB Plant Health and Bio-input Research Center belonging to the University of
\end{abstract}




\section{Como citar:}

FLÓREZ-DELGADO, Dixón Fabian. et al. Comportamiento agronómico y productivo de Tithonia diversifolia en trópico alto, bajo dos esquemas de fertilización. En: Revista Facultad Ciencias Agropecuarias - FAGROPEC Universidad de la Amazonia, Florencia Caquetá. Volumen 11 julio-diciembre, 2019. Pp. 72-78 ISSN-Revista en Línea: 2539-178X
Pamplona. For this, 50 plants were established for each treatment: chemical fertilization, organic fertilization and control under a randomized design after soil analysis and application of corrective measures. For the evaluation of the agronomic and productive variables of plant height, number of stems, number of leaves, weight of stem, weight of leaf, relation leaf - stem, leaf area and dry matter production, descriptive statistical tests were applied, analysis of variance and analysis of separation of means using the Tukey test with a significance of $5 \%$. Statistically significant difference $p<0.05$ was presented for the variables of height, number of leaves and number of stems, with chemical fertilization being the best treatment, while leaf area was higher in organic fertilization. Similar behavior was obtained for the other variables. It is concluded that chemical fertilization influences some agronomic parameters of $T$. diversifolia.

Key words:

Fertilization; Dry matter; Tithonia diversifolia; Agronomic variables.

\section{INTRODUCCIÓN}

En Colombia, los recursos forrajeros son abundantes e incluso desconocidos en cuanto a sus características, comportamiento y usos, por lo cual no son empleados de manera adecuada y eficiente para satisfacer las necesidades del sector ganadero en constante crecimiento (Boschini et al. 2000). La producción de especies forrajeras está enfocada principalmente en la actividad ganadera, y la información reportada en cuanto a existencia, área sembrada, productividad y calidad nutricional es limitada. Debido a la variedad de pisos térmicos y biodiversidad, Colombia presenta un gran potencial en cuanto a la disponibilidad y variedad de recursos forrajeros, los cuales incluyen una gran variedad de especies (Flórez, 2017). Las especies forrajeras existentes tienen como uso principal la alimentación animal, especialmente la ganadería bovina (Xu et al. 2011). Por lo tanto, para este uso específico, se hace importante que la especie forrajera cumpla con características como que sea perenne, fácil rebrote, buena adaptación, productividad de materia seca elevada, fácil establecimiento, resistencia a plagas y enfermedades y tener capacidad de competir con otras especies vegetales no deseadas (Cardona et al. 2012).

Bajo este contexto, los forrajes constituyen la alternativa alimenticia más importante en los sistemas de producción bovina, ya que constituyen la fuente más económica para satisfacer los requerimientos de los hatos bovinos (Flórez y Gómez, 2015). Sin embargo, el desconocimiento en su manejo agronómico y las altas cargas animales han ocasionado degradación de las praderas (Portillo, et al. 2019). La evaluación y selección de forrajes, requiere de estudios que permitan conocer el comportamiento de cada una de las variedades en condiciones climáticas, de manejo y de producción propias de cada región, y de esta manera llevar al sector ganadero a la competitividad y productividad esperada (Cadena et al. 2019).

De esta manera, la $T$. diversifolia se muestra como una alternativa forrajera de gran importancia para las ganaderías gracias a sus características productivas, nutricionales y de adaptación en pro de potenciar los sistemas de producción. En este sentido, el objetivo de este estudio fue evaluar el comportamiento agronómico y productivo de Tithonia diversifolia en trópico alto bajo dos esquemas de fertilización. 


\section{Materiales y métodos}

Lugar de la investigación: esta investigación se desarrolló en el Centro de Investigación en Sanidad Vegetal y Bioinsumos CISVEB perteneciente a la Universidad de Pamplona. Tiene una altitud de 2245 m s.n.m.; con $14^{\circ} \mathrm{C}$ de temperatura promedio, topografía irregular y precipitación anual de $1800 \mathrm{~mm}$.

Material vegetal: para la investigación se definió como material vegetal la Tithonia diversifolia (botón de oro, falso girasol), debido a su adaptación a diferentes pisos térmicos, gran producción de biomasa y excelente calidad nutricional.

Diseño experimental: se utilizó un diseño aleatorizado con dos tratamientos, un testigo y 50 repeticiones. A cada una de las variables agronómicas de la especie forrajera Tithonia diversifolia, se le realizaron pruebas de estadística descriptiva, análisis de varianza y análisis de separación de medias mediante la prueba Tukey $(\mathrm{P}<0.05)$ para determinar las diferencias entre los esquemas de fertilización.

Procedimiento experimental: la investigación inició con la identificación de las propiedades físicas y químicas del suelo. Para ello se tomó una muestra de suelo y se remitió a laboratorio para su análisis. Se tomaron doce submuestras en cuadrícula a una profundidad de $30 \mathrm{~cm}$ (Sosa, 2012). Una vez realizado el análisis de suelo, se adecuó el terreno para la siembra. Se estableció un semillero, con estacas provenientes de plantas sanas y vigorosas con mínimo tres yemas y $30 \mathrm{~cm}$ de longitud. Una vez las plántulas alcanzaron un mínimo de tres hojas fueron llevadas al terreno para su establecimiento definitivo con una distancia de siembra de $50 \mathrm{~cm}$.

Se tomaron las medidas de altura de las plantas, número de hojas, número de tallos, peso de las hojas, área foliar (cada ocho días hasta alcanzar el momento del corte), peso de los tallos, peso de las hojas y relación tallo - hojas (en el momento del corte), que según (Guatusmal, et al. 2020) es cuando el cultivo alcanza el 10\% de la floración, el cual se realizó a $15 \mathrm{~cm}$ del suelo para favorecer el rebrote (Jama, et al. 2000). La producción de biomasa, se estimó a través de un aforo y proyectado a una hectárea base materia seca.

Toma de datos: en la investigación, los datos y mediciones se realizaron de la siguiente manera:

\section{Altura de la planta.}

Se medió con una cinta métrica, partiendo de la base del tallo hasta la hoja más alta, a todas las plantas en cada uno de los tratamientos (Londoño et al. 2018)

Número de hojas y número tallos por planta.

Se realizó a través de conteo manual planta por planta en cada tratamiento.

Peso de hojas y peso de tallos.

Se realizó a través de la recolección del material, siendo llevado a una balanza digital. Se realizó en el momento del corte.

Relación tallo-hojas.

La relación tallo - hojas se midió teniendo en cuenta el cociente del peso de las hojas y el peso del tallo en el momento del corte (Medina et al. 2009). Se calculó teniendo en cuenta la siguiente 
ecuación (1): $H: T=H \underset{!}{W} T$

Dónde:

$\mathrm{H}: \mathrm{T}=$ Relación hoja tallo

$\mathrm{H}=$ Peso seco del componente hoja (Kg MS / ha)

$\mathrm{T}=$ Peso seco del componente tallo (Kg MS / ha)

Área foliar.

Para estimar el índice e área foliar, se empleó la siguiente ecuación(2) (Morales et al. 2006):

$I A F=A F * D P \frac{\square}{=} 10000 \mathrm{~cm}^{2}$

Dónde:

$\mathrm{IAF}=$ índice de área foliar, $\mathrm{AF}=$ área foliar por planta $\left(\mathrm{cm}^{2}\right)$ y $\mathrm{DP}=$ número de plantas $\mathrm{m}^{2}$.

Producción de biomasa.

Para la estimación de la producción de biomasa, se procedió a cortar todo el material vegetal de cada una de las plantas a una altura de $15 \mathrm{~cm}$, procediendo a realizar su pesaje y proyectando a una hectárea. Éste valor se estimó en materia seca.

\section{Modelo estadístico}

Para la presente investigación, se empleó un diseño aleatorizado bajo el siguiente modelo matemático:

$$
Y h i j=\mu+T t o i+\epsilon i j
$$

$\mathrm{Y}_{\mathrm{ij}}=$ Variables agronómicas y producción de biomasa.

$\mu=$ Promedio poblacional.

$\mathrm{Tto}_{\mathrm{i}}=$ Efecto de los tratamientos.

$€_{\mathrm{ij}}=$ Error experimental.

Se realizó un análisis de varianza de un factor: esquema de fertilización con una significancia del 5\%, aplicando supuestos de Normalidad y Homogeneidad de varianzas para evaluar el efecto de los tratamientos sobre estas variables agronómicas y de producción de biomasa.

\section{Resultados y análisis}

Como resultados del análisis de laboratorio, se obtuvo un suelo fuertemente ácido con un $\mathrm{pH}$ de 5,48, además de niveles bajos de materia orgánica, fósforo, magnesio y aluminio con valores de $3,82 \mathrm{ppm}, 0,05 \mathrm{Meq} / 100 \mathrm{~g}$, $0,89 \mathrm{Meq} / 100 \mathrm{~g}$ y $0,0004 \mathrm{Meq} / 100 \mathrm{~g}$ respectivamente. El potasio se presentó en una concentración media con valores de $0,32 \mathrm{Meq} / 100 \mathrm{~g}$. La textura fue clasificada como franco limoso, con 0,029\% de saturación de aluminio y $65,67 \%$ de saturación de bases, siendo ésta última adecuada sin presencia altas concentraciones de $\mathrm{Al}++$ por lo que no fue necesario aplicar ningún tipo de enmienda. Para cubrir los requerimientos de fósforo y nitrógeno se aplicaron $145 \mathrm{~kg}$ de DAP por hectárea año y para el potasio $49 \mathrm{~kg}$ de $\mathrm{KCl}$ para mantenimiento, esto para el esquema de fertilización químico, mientras que para el esquema orgánico se empleó lombrihumus en una relación de $440 \mathrm{~kg}$ por hectárea año.

En la tabla 1, se muestran los resultados del análisis de varianza realizado a las variables agronómicas y de producción de biomasa de la Tithonia diversifolia bajo dos esquemas de fertilización.

Para las variables agronómicas de altura de planta, número de hojas, número de tallos y área foliar, se presentó comportamiento estadísticamente diferente $(\mathrm{p}<0,05)$, siendo la mejor media en los tratamientos de fertilización química para las tres primeras variables, mientras que para área foliar la fertilización orgánica presentó el mejor tenor. En las demás variables: peso de tallo, peso de hoja, relación hoja:tallo y productividad 
Tabla 1. Variables agronómicas y de producción de biomasa de la Tithonia diversifolia bajo dos esquemas de fertilización.

\begin{tabular}{lcccccccc}
\hline & & \multicolumn{2}{c}{ Testigo } & \multicolumn{2}{c}{ Tratamiento 1 } & \multicolumn{3}{c}{ Tratamiento 2 } \\
Variable & Unidad & Media & E.E. & Media & E.E. & Media & E.E. & p-valor \\
\hline Altura planta & $\mathrm{cm}$ & 52,42 & $\pm 2,96 \mathrm{a}$ & 58,94 & $\pm 2,68 \mathrm{~b}$ & 33,43 & $\pm 1,99 \mathrm{c}$ & 0 \\
Número de hojas & Unidad & 110,52 & $\pm 4,03 \mathrm{a}$ & 130,38 & $\pm 5,50 \mathrm{~b}$ & 46,83 & $\pm 4,55 \mathrm{c}$ & 0 \\
Número de tallos & Unidad & 7,54 & $\pm 0,69 \mathrm{a}$ & 8,01 & $\pm 0,51 \mathrm{~b}$ & 3,86 & $\pm 0,35 \mathrm{c}$ & 0 \\
Área Foliar & - & 3,4 & $\pm 0,12 \mathrm{a}$ & 3,19 & $\pm 0,08 \mathrm{~b}$ & 3,68 & $\pm 0,13 \mathrm{c}$ & 0,015 \\
Peso Tallo & $\mathrm{Kg}$ & 1,24 & $\pm 0,26 \mathrm{a}$ & 1,88 & $\pm 0,46 \mathrm{a}$ & 1,86 & $\pm 0,50 \mathrm{a}$ & 0,543 \\
Peso Hoja & $\mathrm{Kg}$ & 0,84 & $\pm 0,17 \mathrm{a}$ & 1,04 & $\pm 0,21 \mathrm{a}$ & 1,12 & $\pm 0,17 \mathrm{a}$ & 0,267 \\
Relación Hoja:Tallo & - & 0,67 & $\pm 0,07 \mathrm{a}$ & 0,57 & $\pm 0,04 \mathrm{a}$ & 0,61 & $\pm 0,08 \mathrm{a}$ & 0,13 \\
Biomasa & $\mathrm{Kg}$ & 2,88 & $\pm 0,41 \mathrm{a}$ & 3,62 & $\pm 0,66 \mathrm{a}$ & 3,88 & $\pm 0,62 \mathrm{a}$ & 0,345 \\
\hline
\end{tabular}

Letras diferentes en las filas indican diferencia estadísticamente significativa $(\mathrm{p}<0,05)$

expresada en biomasa seca, no se presentó diferencia estadísticamente significativa $(\mathrm{p}>0,05)$.

Botero et al. (2019), afirman que la fertilización química genera un impacto importante sobre el comportamiento agronómico de la T. diversifolia, especialmente en las variables de altura de planta, número de tallos, peso del tallo y peso de la hoja con dosis crecientes de nitrógeno, concordando con los resultados de la presente investigación. Estos mismos autores, reportan datos de altura de planta en un rango que oscila entre los $90,65 \mathrm{~cm}$ y los $154,88 \mathrm{~cm}$, relación hoja:tallo en un rango de 0,79 a 1,46 y tallos por planta entre 14 y 29 siendo superiores a los datos obtenidos en el presente estudio. Estas variables, tienen relación estrecha con la producción de biomasa del forraje y finalmente con la capacidad de carga de la pradera, así como con la palatabilidad y el nivel de consumo por parte de los animales. El número de hojas es una característica importante en el momento de evaluar un forraje por su productividad, dado que está directamente relacionada con la disponibilidad de alimento especialmente en épocas de escasez (López et al. 2019). Como resultado en esta investigación, se obtuvo un mayor número de hojas bajo el esquema de fertilización química con 130 por planta respecto a la fertilización orgánica y a la ausencia de la misma. Las variables peso de hoja y peso de tallo presentaron comportamiento estadísticamente similar, siendo superiores a los reportados por Holgín et al. (2015) con $0,52 \mathrm{~kg}$ y $0,43 \mathrm{~kg}$ para hojas y tallos respectivamente y a los obtenidos por Gallego et al. (2017) con $0,58 \mathrm{~kg}$ para hojas y $0,62 \mathrm{~kg}$ para tallos.

La aplicación de abonos orgánicos aporta gran cantidad de nutrientes, facilita la formación de compuestos y la activación de muchos procesos biológicos en el suelo (López et al. 2019), que junto con la capacidad de la $T$. diversifolia de extraer y absorber nutrientes a través de su sistema radicular, permite un desarrollo adecuado de este forraje (Gallego et al. 2014), presentando grandes porcentajes de sobrevivencia favoreciendo el establecimiento. Este tipo de fertilización, tiene gran importancia en el desarrollo del área foliar de los forrajes especialmente, conllevando a una mayor producción de biomasa representada en materia seca. Esta variable agronómica, juega un papel importante en el establecimiento y desarrollo de T. diversifolia debido a su asocio con procesos fisiológicos, fotosintéticos y de balance de energía (Gallego et al. 2017).

La producción de biomasa, es un factor clave que direcciona la extracción de nutrientes del suelo y los esquemas de fertilización adquieren gran importancia en especies forrajeras con gran potencial productivo (Ramírez, 2018). Para identificar la dosis apropiada de fertilizante se debe tomar en cuenta el nivel esperado de producción de forraje, las condiciones del suelo, el ambiente, la tecnología aplicada y el potencial genético de productividad de la especie (Bernal y Espinosa, 2003). La producción de biomasa es afectada por factores climáticos, edáficos y especialmente por prácticas agronómicas como fertilización, densidad de siembra, 
riego y control de arvenses (Mahecha y Rosales, 2005). La producción de biomasa seca de T. diversifolia oscila en un rango de 30 a $70 \mathrm{t} / \mathrm{ha} /$ corte (Medina et al. 2009), estando los resultados de la presente investigación acordes a ello, con $31 \mathrm{t} / \mathrm{ha}$ /corte bajo la fertilización orgánica, y siendo superiores a los reportados por Téllez y Mendoza (2014) con 26,6 t/ha/corte y Navas y Montaña (2019) con una media de 25,5 t/ha/corte de materia seca. Los abonos orgánicos, tienen influencia sobre la disponibilidad de minerales en el suelo, incorporándose a gran velocidad y permitiendo que los nutrientes sean asimilados por el sistema radicular de las plantas para su crecimiento, dando como resultado la producción de biomasa verde. López y Miranda (2015) exponen que los abonos orgánicos ejercen efectos positivos en la estructura del suelo, mejorando la retención de agua, la permeabilidad, la textura, la aireación y oxigenación, facilitando de esta manera que el sistema radicular de la $T$. diversifolia obtenga los nutrientes necesarios para su establecimiento y desarrollo.

\section{Conclusiones}

La fertilización química de la Tithonia diversifolia tiene influencia sobre las variables agronómicas de altura de planta, número de hojas y número de tallos, mientras que los abonos orgánicos favorecen la variable área foliar. Las demás variables agronómicas de peso de hoja, peso de tallo, relación hoja tallo y de productividad expresada en biomasa seca no son afectadas por el tipo de fertilización.

\section{Literatura citada}

BERNAL, J., \& ESPINOSA, J. Manual de nutrición y fertilización de pastos. Potash and Phosphate Institute of Canada. 94p. 2003.

BOSCHINI, C., DORMOND, H., \& CASTRO, A. Composición química de la morera (Morus alba), para uso en la alimentación animal: densidades y frecuencias de poda. Agronomía mesoamericana: 11, Pp. 41-49. 2000 .

CADENA, M., GARCÍA, M., \&. CASTRO, E. Estabilidad fenotípica de genotipos de Lolium sp. en el trópico alto de Nariño, Colombia. Agronomía. Mesoamericana. 30 (2):483-495, 2019. \{En línea\}. Disponible en: https://cutt.ly/0yRdeiF

CARDONA, E., RÍOS, L., \& PEÑA, J. Disponibilidad de Variedades de Pastos y Forrajes como Potenciales Materiales Lignocelulósicos para la Producción de Bioetanol en Colombia. Información tecnológica, 23 (6): 87-96. 2012.\{En línea\}. Disponible en: https://cutt.ly/WyRs3b7

FLÓREZ, D. Estimación de la capacidad de carga del sistema de producción lechero de la vereda Fontibón del municipio de Pamplona. Mundo Fesc, 13, 15-21.2017.

FLÓREZ, D., \& GÓMEZ, B. Estimación de costos de producción de terneras en fase de cría en la hacienda aposentos, municipio de Chinácota, Norte de Santander. Revista Facultad Ciencias Agropecuarias FAGROPEC. Universidad de la Amazonia, Florencia-Caquetá. 8 (2): Pp. 88-90. 2015

GALLEGO, L., MAHECHA, L., \& ANGULO, J. Calidad nutricional de Tithonia diversifolia Hemsl. A Gray bajo tres sistemas de siembra en el trópico alto. Revista Agronomía Mesoamericana, 28(1), Pp. 213-22. 2017

GALLEGO, L., MAHECHA, L., \& ANGULO, J. Potencial forrajero de Tithonia diversifolia Hemsl. A Gray en la producción de vacas lecheras. Revista Agronomía Mesoamericana, 25 (2), Pp. 393-403. 2014

GUATUSMAL, C., ESCOBAR, L., MENESES, D., CARDONA, L., \& CASTRO, E. Producción y calidad de 
Thithonia diversifolia y Sambucus nigra en trópico altoandino colombiano. Agronomía Mesoamericana, 31 (1): Pp. 193-208. 2020

HOLGUÍN, V., ORTIZ, S., VELASCO, N., \& MORA, J. Evaluación multicriterio de 44 introducciones de Tithonia diversifolia (hemsl.) A. Gray en Candelaria, Valle del Cauca. Revista de la Facultad de Medicina Veterinaria y de Zootecnia, 62 (2):57-72, 2015

JAMA, B., PALM, C., BURESH, R., NIANG, A., GACHENGO, C., NZIGUHEBA, G., \& AMADALO, B. Tithonia diversifolia as a green manure for soil fertility improvement in western Kenya A review. Agrofor. Syst. 49,Pp. 201-221, 2000

LONDOÑO, J., MAHECHA, L., \& ANGULO, J. Desempeño agronómico y valor nutritivo de Tithonia diversifolia (Hemsl.) A Gray para la alimentación de bovinos. Revista Colombiana de Ciencia Animal, 11 (1). 2018.

LÓPEZ,A., DÍAZ, L., \& CALERO, W. Efecto de tres fertilizantes orgánicos en el crecimiento de botón de oro en condiciones de vivero, Nueva Guinea, RACCS, 2017. Revista Ciencia e Interculturalidad, 12 (24): 203 214. Enero-junio, 2019.

LÓPEZ, G., \& MIRANDA, J. Producción y calidad de forraje en abonos orgánicos en Brachiaria brizanta, Finca Regalo de Dios, Yolaina Nueva Guinea. 2015.

MAHECHA, L., \& ROSALES, M. Valor nutricional de follaje de botón de oro Tithonia diversifolia (Hemsl.) Gray, en la producción animal en el trópico. Livestock Res Rural Develop, 17 (9), 2005.

MEDINA, M., GARCÍA, D., GONZÁLEZ, M., COVA, L., \& MORATINOS, P. "Variables morfoestructurales y de calidad de la biomasa de Tithonia diversifolia en la etapa inicial de crecimiento". Zootecnia Tropical, 27 (2): 121-134, 2009.

MORALES, A., JIMÉNEZ, V., VELASCO, V., VILlEGAS, A., ENRÍQUEZ, V., \& HERNÁNDEZ, Y. "Evaluación de 14 variedades de alfalfa con fertirriego en la mixteca de Oaxaca". Técnica Pecuaria en México, Vol. 44, no. 3,pp. 277-288, 2006.

NAVAS, A., \& MONTAÑA, V. Comportamiento de Tithonia diversifolia bajo condiciones de bosque húmedo tropical. Revista de Investigación Veterinaria, Perú, 30(2): Pp. 721-732, 2019.

PORTILLO, P., MENESES, D., MORALES, S., CADENA, M., \& CASTRO, E. Evaluación y selección de especies forrajeras de gramíneas y leguminosas en Nariño, Colombia. Pastos y Forrajes, 42 (2): Pp. 93-103. 2019

RAMÍREZ, R. Extracción de nutrientes y productividad del botón de oro (Tithonia diversifolia) con varias dosis de fertilización nitrogenada. Revista InterSedes, 19 (39), enero-junio 2018.

SOSA, D. Cómo realizar un muestreo de suelos. INTA. Argentina, 2012.

TÉLLEZ, S., \& MENDOZA, R. Comportamiento productivo de Tithonia diversifolia en bancos forrajeros, bajo condiciones de suelos de piedemonte llanero. Tesis de Zootecnista. Bogotá: Univ. de la Salle. Pp. 47. 2014.

XU, J., WANG, Z., \& CHENG, J. Bermuda grass as feedstock for biofuel production: a review, Bioresource technology: 102 (17): Pp.7613,7620.2011 